\title{
Capacidade funcional de idosos restritos ao domicílio, do conjunto Ruy Virmond Carnascialli, Londrina/PR
}

\author{
Functional capacity of elderly who are forced to stay at home due to \\ their physical limitations, from the housing development \\ Ruy Virmond Carnascialli, Londrina/PR
}

\author{
Celita Salmaso Trelha ${ }^{1}$; Tatiana Nakaoski ${ }^{2}$; Simone Silveira Franco ${ }^{2}$; Mara Solange \\ Gomes Dellaroza ${ }^{3}$; Kiyomi Nakanishi Yamada ${ }^{3}$; Marcos Cabrera ${ }^{3}$; Arthur Eumann \\ Mesas $^{3}$; Carlos César Benes Gaetan ${ }^{3}$
}

\section{Resumo}

\begin{abstract}
A incapacidade funcional é definida pela presença de dificuldade no desempenho de atividades da vida cotidiana ou mesmo pela impossibilidade de desempenhá-las. O objetivo do estudo foi avaliar a incapacidade funcional de idosos restritos ao domić́lio. Participaram deste estudo 104 idosos acima de 60 anos, da área de abrangência da Unidade Básica da Saúde(UBS) do Conjunto Habitacional Ruy Virmond Carnascialli, Região Norte da cidade de Londrina/PR. Para a coleta de dados, foi utilizado um questionário sócio-demográfico e o Functional Autonomy Measurement System, instrumento composto por 29 aspectos divididos em cinco domínios: Atividade de Vida Diária, Mobilidade, Comunicação, Função Mental e Atividade Instrumental de Vida Diária. Para o tratamento estatístico dos dados, foi utilizado o programa Epi Info 6.04b e software Excel da Microsoft. A amostra constituiu-se predominantemente por idosos do gênero feminino e com média de idade de 75,8 $\pm 8,1$ anos. Em relação às atividades de vida diária, os idosos mostraram-se dependentes principalmente no banho $29(27,9 \%)$, no cuidado pessoal $22(21,1 \%)$ e higiene $22(21,1 \%)$.No domínio mobilidade a maior incapacidade foi em relação a locomoção externa 28 (26,9\%). A visão foi o item mais comprometido entre os idosos, sendo que $60(57,7 \%)$ referiram ter dificuldade ou precisarem de auxílio/supervisão. Já em relação à atividade instrumental de vida diária, fazer compras e utilizar o telefone foram as atividades que obtiveram a maior porcentagem de dependência, $53,8 \%$ e $42,3 \%$ respectivamente. Os resultados mostraram a necessidade de estratégias efetivas para a prevenção e reabilitação do declínio funcional.

Palavras-chave: Idosos. Incapacidade funcional. Domicílio. Atividades de vida diária. Atividades instrumentais.
\end{abstract}

\footnotetext{
${ }^{1}$ Fisioterapeuta, Docente do Departamento de Fisioterapia da Universidade Estadual de Londrina, Mestre em Saúde Coletiva. Doutoranda em Medicina e Ciências da Saúde.Rua Alagoas 1110, apto 303 - CEP 86020-430 - Centro - Londrina PR Fone: (43) 33269289 -E-mail: celita@uel.br

2 Acadêmicas do Curso de Fisioterapia da Universidade Estadual de Londrina e Bolsistas de Extensão do Projeto PAINP

3 Docentes e profissionais participantes do Projeto PAINP
} 


\begin{abstract}
The functional incapacity is defined by the difficulty in developing daily life activities or even the impossibility of performing them. The purpose of the study was to analyze the functional incapacity of elderly who are forced to stay at home due to their physical limitations. A hundred and four individuals above the age of 60 years old, participated in this study. All of them are from the inclusion area of the UBS ( Health Basic Unit) from the Housing Development Ruy Virmond Carnascially, northern area of Londrina/PR. As for the data gathering, it was used a demographic questionnaire and the Functional Autonomy Measurement System, an instrument which consists of 29 aspects divided in five skills: Daily Life Activity; Mobility; Communication; Mental Function and Instrumental Daily Life Activity. For the statistical treatment it was used the program Epi Info 6.04b and the software Excel of Microsoft. The sample consisted predominantly of elderly women with an average age of 75,8 to 81 years old. In relation to the daily life activities, the elderly showed to be dependent mainly in the bath: $29(27,9 \%)$, in their personal care: $22(21,1 \%)$, and hygiene: $22(21,1 \%)$. In terms of mobility, $28(26,9 \%)$ mentioned incapacity in relation to walking outside. The eyesight was considered the biggest problem among the elderly, 60 $(57,7 \%)$ mentioned to have difficulties and needed aid/supervision. In relation to instrumental daily life activity, to go shopping and using the telephone obtained the highest percentage of dependence: $53,8 \%$ and $42,3 \%$, respectively. The results showed the need of effective strategies for the prevention and rehabilitation of the functional decline.
\end{abstract}

Key words: The elderly. Functional incapacity. Home. Daily life activities. Instrumental activities.

\section{Introdução}

O envelhecimento é um processo dinâmico e progressivo no qual ocorrem alterações físiológicas, morfológicas, funcionais e bioquímicas que vão alterando progressivamente o organismo, tornandoo mais susceptível a agressões (FEDRIGO, 1999).

A capacidade funcional representa a independência do indivíduo viver realizar suas atividades físicas e mentais necessárias para manutenção de suas atividades básicas e instrumentais, ou seja: tomar banho, vestir-se, realizar higiene pessoal, transferir-se, alimentar-se, manter a continência, preparar refeições, controlar as finanças, tomar medicamentos, arrumar a casa, fazer compras, usar transporte coletivo, usar telefone e caminhar uma certa distância (RAMOS, 2003; SOUZA; IGLESIAS, 2002).

A incapacidade funcional é a inabilidade de manter as atividades físicas e/ou mentais necessárias a uma vida independente e autônoma (PARANÁ, 2004a). Esse declínio funcional ocorre na população idosa, com sintomas inespecíficos, apresentação atípica, curso insidioso, e engloba manifestações físicas, psicológicas, sociais e funcionais. O declínio funcional é identificado progressivamente pela perda da capacidade de executar as atividades básicas de vida diária (ABVDs) e as atividades instrumentais de vida diária (AIVDs). Fisicamente, o idoso pode referir fadiga, fraqueza, perda de apetite, adipsia, perda de peso, quedas e incontinências. Já os sintomas psicológicos relatados são perda de atenção, interesse, iniciativa e motivação. Algumas vezes, pode se notar declínio cognitivo. Além disso, socialmente, o idoso se afasta de atividades usuais e fica mais isolado. A negligência com os cuidados da casa e com os cuidados pessoais podem ser observados (HÉBERT, 1997).

A grande maioria dos idosos desenvolve, ao longo da vida, doenças crônicas, decorrentes da perda contínua da função de órgãos e sistemas biológicos. Essa perda de função pode levar a limitações funcionais, e, por sua vez, pode gerar incapacidade, conduzindo à dependência de pessoas ou de equipamentos específicos para a realização de tarefas essenciais à sobrevivência no dia a dia (PARANÁ, 2004a).

Percebe-se que, nas alterações relacionadas com a idade, estão a presença de fatores de risco e a ocorrência de doenças crônico-degenerativas, que determinam para o idoso o grau de dependência, 
relacionado diretamente com a perda da autonomia e dificuldade de realizar as atividades básicas e instrumentais da vida diária, interferindo na qualidade de vida do idoso (CHAIMOWICZ, 1996).

As causas do declínio funcional estão relacionadas, principalmente, a: doenças como infarto, infecções, acidente vascular encefálico, parkinson, insuficiência renal, hipotireoidismo, tuberculose e câncer; descompensação de uma condição crônica (diabete, hepatite, insuficiência renal); ou crise social ou psicológica associada à morte de cônjuge, admissão no hospital ou transferência para instituição e depressão (HÉBERT, 1997).

De acordo com Cordeiro (2002) a perda da capacidade funcional está associada a predição de fragilidade, dependência, institucionalização, risco aumentado de quedas, morte e problemas de mobilidade, trazendo complicações ao longo do tempo, e gerando cuidados de longa permanência e alto custo em virtude da necessidade de assistência médica e risco de hospitalização, contribuindo significativamente para a atual crise no sistema de saúde (BENNET, 1998).

No Paraná, os idosos acima de 60 anos representam 7,61\% da população do Estado. Contudo, eles foram responsáveis por $21 \%$ das internações realizadas pelo SUS em 2000 (Janeiro Outubro), utilizando $26 \%$ dos recursos destinados a este fim (PARANÁ, 2004b).

Além disso, a família freqüentemente fica esgotada, e uma crise familiar pode levar à violência e até pensamentos de internar o idoso em instituições geriátricas. Este fenômeno foi chamado de "dumping", que significa abandono. Muitas vezes, uma decorrência dessa situação é o fato de considerar-se, erroneamente, que os pacientes com declínio funcional apresentam problemas sociais. Tudo isso ocorre devido à exaustão familiar, que freqüentemente acompanha o declínio funcional do idoso. Em 1998, de 100 casos consecutivos de "dumping", observou-se que a causa do declínio funcional era física ou psicológica e que o componente social era mais uma conseqüência do que uma causa da síndrome (HÉBERT, 1997).

Estudos populacionais revelam que cerca de $40 \%$ dos idosos de 65 anos ou mais necessitam de algum tipo de ajuda para realizar suas atividades da vida diária, como cuidar das finanças, preparar refeições e limpar a casa, e uma parcela menor, mas significativa de $10 \%$, requer auxílio para realizar tarefas básicas, como tomar banho, vestir-se, ir ao banheiro, alimentar-se e até, sentar e levantar de cadeiras e camas (RAMOS et al., 1993).

Bennet (1998) afirma que cerca de 5,3\% dos adultos com 65 a 75 anos necessitam de assistência para realizar as atividades básicas do quotidiano (tomar banho, vestir-se, caminhar, uso de sanitários e transferir-se do leito para uma cadeira). Pouco menos de $6 \%$ necessitam de auxílio para as atividades práticas do quotidiano (cozinhar, fazer compras, usar o telefone, serviços domésticos e controle das finanças pessoais). Já aos 85 anos de idade, esses índices aumentam dramaticamente para $35 \%$ e $40 \%$ respectivamente.

Em Londrina, o fenômeno do envelhecimento populacional já é percebido quando comparados os dados do censo de 1960 com os de 2000. No primeiro, a população acima de 69 anos correspondia a 3,41\%, elevando-se para $9,34 \%$ no censo de 2000 , o que corresponde a 41.780 pessoas idosas. Esta população, em sua grande maioria, apresenta um nível sócio econômico precário e vivência dificuldades de acesso a Serviços de Saúde que lhes proporcionem uma atenção integrada, ou seja, que contemplem os aspectos bio-psico sociais da velhice.

Diante dessa realidade, encontra-se em vigência o Projeto de Assistência Interdisciplinar ao Idoso em Nível Primário (PAINP), um Projeto de Extensão da Universidade Estadual de Londrina. Nesse projeto, um grupo de docentes e profissionais dos cursos de graduação em medicina, enfermagem, fisioterapia, odontologia e serviço social desenvolvem atividades envolvendo a instituição formadora (docentes e alunos), o serviço público de saúde do 
Conjunto Habitacional Ruy Virmond Carnascialli, representado pela Unidade Básica de Saúde (UBS) e equipes do Programa de Saúde da Família (PSF) do local, e a comunidade alvo, composta por todos os idosos e cuidadores formais e informais residentes na área de abrangência do estudo. A escolha do conjunto residencial obedeceu aos seguintes critérios: número elevado de idosos na área, equipe profissional da UBS motivada para o trabalho com idosos; população de diferentes níveis econômicos, incluindo áreas carentes e de nível médio e população motivada e já pré-organizada.

A revisão de literatura não revelou nenhum estudo que considerasse a capacidade funcional de idosos restritos ao domicílio e portanto esse diagnóstico das necessidades desses idosos permite um direcionamento no planejamento e na implantação de estratégias e políticas de saúde.

Este estudo tem por objetivo avaliar o nível de capacidade funcional dos idosos restritos ao domicílio do Conjunto Habitacional Ruy Virmond Carnascialli, da cidade de Londrina/PR.

\section{Material e Método}

Participaram deste estudo 104 idosos acima de 60 anos restritos ao ambiente domiciliar, na área de abrangência da UBS do Conjunto Habitacional Ruy Virmond Carnascialli, Região Norte de Londrina.

Os critérios de inclusão utilizados foram: idade igual ou superior a 60 anos; ser morador do Conjunto Habitacional Ruy Virmond Carnascialli, no Município de Londrina (PR) e estar incluído no Programa Saúde da Família (PSF) da Unidade Básica de Saúde (UBS) local.

A condição de "restrito ao domicílio" não está relacionada necessariamente a um alto nível de dependência, mas a uma ou mais incapacidades (físicas ou mentais) que resultem em limitações de deslocamento independente para fora do domicílio.

Para a coleta de dados, foi realizada entrevista estruturada abordando aspectos sócio demográficos e presença de dor e doenças e avaliação do grau de incapacidade. Esta última foi avaliada através do Functional Autonomy Measurement System (HÉBERT et al., 2001), instrumento composto por 29 aspectos divididos em cinco domínios: Atividade de Vida Diária, Mobilidade, Comunicação, Função Mental e Atividade Instrumental de Vida Diária. Considerou-se, para cada tarefa funcional questionada nesse instrumento três categorias de respostas possíveis: independente, apresenta dificuldade ou realiza com auxílio ou supervisão e totalmente dependente.

As entrevistas foram realizadas por acadêmicos de fisioterapia, enfermagem, medicina e odontologia, participantes do Projeto PAINP sob orientação dos docentes envolvidos, em visitas domiciliares agendadas previamente por parte do agente comunitário de saúde. Nos casos de idosos com afasia, alteração relacionada a memória ou confusão mental os cuidadores foram os informantes.

O Projeto PAINP e o Termo de Consentimento Livre e Esclarecido foram submetidos ao Comitê de Ética em Pesquisa da Universidade Estadual de Londrina, com parecer favorável.

A Análise estatística foi descritiva e consistiu na organização e descrição dos dados, na identificação de valores e na quantificação da variabilidade presente nos dados (SOARES; SIQUEIRA, 1999). Para o tratamento estatístico dos dados foi utilizado o programa Epi Info $6.04 \mathrm{~b}$ e software Excel da Microsoft.

\section{Resultados}

Dos 104 idosos estudados 70 (67,3\%) eram do sexo feminino e $34(32,7 \%)$ do sexo masculino. A média de idade encontrada foi de 75,8+8,1, sendo a menor idade de 60 anos e a maior de 96 anos. Dos 102 idosos que relataram a idade, $68(66,7 \%)$ apresentaram idade entre 60 a 80 anos e $34(33,3 \%)$ acima de 80 anos, como pode ser observado na Figura 1. 


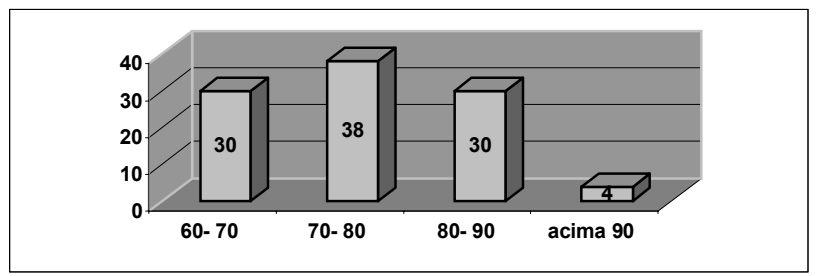

*AVC (acidente vascular cerebral)

Figura 1. Distribuição dos idosos restritos ao domicílio segundo a faixa etária em anos. Londrina, 2004

Em relação ao estado civil, verificou-se que 51 $(49,0 \%)$ referiram ser viúvos, $45(43,3 \%)$ casados, 5 $(4,8 \%)$ separados e $2(1,9 \%)$ solteiros.

O grupo de idosos não alfabetizados foi predominante 44 (42,3\%), seguida dos idosos que não completaram o $1^{\circ}$ grau $29(27,9 \%)$, semi-alfabetizados $18(17,3 \%), 1^{\circ}$ grau completo $7(6,7 \%), 2^{\circ}$ grau completo $2(1,9 \%), 2^{\circ}$ grau incompleto $2(1,9 \%)$ e 2 $(1,9 \%)$ idosos não responderam esta questão. Não foi encontrado idoso com curso superior.

Entre os idosos entrevistados 94 (90,4\%) reportaram apresentar uma renda familiar mensal entre 1 a 4 salários mínimos, 2 idosos $(1,9 \%)$ com renda mensal inferior a 1 salário mínimo e somente 6 (5,8\%) com renda maior de 5 salários mínimos, caracterizando a homogeneidade da população estudada.

Os idosos foram questionados sobre diagnósticos clínicos, responsáveis por incapacidades e observouse que a hipertensão arterial foi o diagnóstico mais freqüente entre os pesquisados $(74,0 \%)$, seguido pelo diabetes $(47,1 \%)$ como pode ser observado na Tabela 1. A taxa de não-resposta em alguns diagnósticos foi alta, chegando a $12(11,5 \%)$ nos diagnósticos "infarto" e "doença de Alzheimer".
Tabela 1 . Distribuição dos idosos restritos ao domicílio segundo o diagnóstico clínico. Londrina, 2004.

\begin{tabular}{|c|c|c|c|c|c|c|c|c|}
\hline \multirow{2}{*}{$\begin{array}{l}\text { Diagnóstico } \\
\text { Clínico }\end{array}$} & \multicolumn{2}{|c|}{ Sim } & \multicolumn{2}{|c|}{ Não } & \multicolumn{2}{|c|}{ Não Sabe } & \multicolumn{2}{|c|}{$\begin{array}{c}\text { Taxa de não- } \\
\text { resposta }\end{array}$} \\
\hline & $\mathbf{n}$ & $\%$ & $\mathbf{n}$ & $\%$ & n & $\%$ & $\mathbf{n}$ & $\%$ \\
\hline$\overline{\mathrm{AVC}}$ & 41 & 39,4 & 55 & 52,9 & 3 & 2,9 & 5 & 4,8 \\
\hline Infarto & 11 & 10,6 & 79 & 76,0 & 2 & 1,9 & 12 & 11,5 \\
\hline Diabetes & 49 & 47,1 & 49 & 47,1 & 3 & 2,9 & 3 & 2,9 \\
\hline Hipertensão & 77 & 74,0 & 22 & 21,1 & 3 & 2,9 & 2 & 1,9 \\
\hline Depressão & 30 & 28,8 & 58 & 55,8 & 8 & 7,7 & 8 & 7,7 \\
\hline Alzheimer & 5 & 4,8 & 73 & 70,2 & 14 & 13,5 & 12 & 11,5 \\
\hline
\end{tabular}

Os idosos foram questionados também acerca da presença de dor nos últimos sete dias precedentes à aplicação do questionário e dos 104 idosos pesquisados, $68(65,4 \%)$ relataram a presença de dor.

A capacidade funcional foi dividida em cinco domínios principais: AVDs, Mobilidade, Comunicação, Estado Mental e Utilização de recursos. Em relação às atividades de vida diária, verificou-se uma maior dependência para as atividades referentes ao banho $29(27,9 \%)$, vestir-se $25(24,0 \%)$, cuidado pessoal $22(21,1 \%)$ e higiene $22(21,1 \%)$ como pode ser observado na Tabela 2.

Tabela 2 . Distribuição dos idosos restritos ao domicílio segundo o grau de incapacidade no domínio: AVDs. Londrina, 2004.

\begin{tabular}{|c|c|c|c|c|c|c|c|c|c|c|}
\hline \multirow[t]{2}{*}{ Atividade } & \multicolumn{2}{|c|}{$\begin{array}{c}\text { Indepen } \\
\text { dente }\end{array}$} & \multicolumn{2}{|c|}{$\begin{array}{c}\text { Dificuldade/ } \\
\text { Supervisão/ } \\
\text { Auxílio }\end{array}$} & \multicolumn{2}{|c|}{$\begin{array}{l}\text { Totalmente } \\
\text { Dependente }\end{array}$} & \multicolumn{2}{|c|}{$\begin{array}{l}\text { Não se } \\
\text { aplica }\end{array}$} & \multicolumn{2}{|c|}{$\begin{array}{l}\text { Taxa de } \\
\text { não- } \\
\text { resposta }\end{array}$} \\
\hline & $\mathbf{n}$ & $\%$ & $\mathbf{n}$ & $\%$ & $\mathbf{n}$ & $\%$ & $\mathbf{n}$ & $\%$ & $\mathbf{n}$ & $\%$ \\
\hline Alimentação & 76 & 73,1 & 15 & 14,4 & 13 & 12,5 & - & - & - & - \\
\hline Higiene & 65 & 62,5 & 16 & 15,4 & 22 & 21,1 & - & - & 1 & 1,0 \\
\hline Vestir-se & 65 & 62,5 & 14 & 13,5 & 25 & 24,0 & - & - & - & - \\
\hline Cuidado & 65 & 62,5 & 10 & 9,6 & 22 & 21,1 & - & - & 7 & 6,7 \\
\hline Cont.Urinária & 65 & 62,5 & 21 & 20,2 & 15 & 14,4 & 2 & 1,9 & 1 & 1,0 \\
\hline Cont. Fecal & 76 & 73,1 & 11 & 10,6 & 15 & 14,4 & 2 & 1,9 & - & - \\
\hline Banho & 64 & 61,5 & 9 & 8,6 & 29 & 27,9 & - & - & 2 & 1,9 \\
\hline
\end{tabular}

Em relação ao domínio mobilidade, verificou-se que os idosos apresentaram maior dependência em locomoção externa $28(26,9 \%)$, escadas $25(24,0 \%)$ e transferências $18(17,3 \%)$ como pode ser observado na Tabela 3. 
Tabela 3. Distribuição dos idosos restritos ao domicílio segundo o grau de incapacidade no domínio Mobilidade. Londrina, 2004.

\begin{tabular}{|c|c|c|c|c|c|c|c|c|c|c|}
\hline \multirow[t]{2}{*}{ Atividade } & \multicolumn{2}{|c|}{ Independente } & \multicolumn{2}{|c|}{$\begin{array}{c}\text { Dificuldade/ } \\
\text { Supervisão/ } \\
\text { Auxílio }\end{array}$} & \multicolumn{2}{|c|}{$\begin{array}{l}\text { Totalmente } \\
\text { Dependente }\end{array}$} & \multicolumn{2}{|c|}{ Não se aplica } & \multicolumn{2}{|c|}{$\begin{array}{l}\text { Taxa de não- } \\
\text { resposta }\end{array}$} \\
\hline & $\mathbf{n}$ & $\%$ & $\mathbf{n}$ & $\%$ & $\mathbf{n}$ & $\%$ & $\mathbf{n}$ & $\%$ & $\mathbf{n}$ & $\%$ \\
\hline Transferência & 52 & 50,0 & 23 & 22,1 & 18 & 17,3 & 2 & 1,9 & 9 & 8,6 \\
\hline Locomoção Interna & 59 & 56,7 & 27 & 26,0 & 16 & 15,4 & - & - & 2 & 1,9 \\
\hline Locomoção Externa & 30 & 28,8 & 38 & 36,5 & 28 & 26,9 & 4 & 3,8 & 4 & 3,8 \\
\hline $\begin{array}{l}\text { Colocação de } \\
\text { Próteses/Orteses }\end{array}$ & 19 & 18,3 & 3 & 2,9 & 4 & 3,8 & 69 & 66,3 & 9 & 8,6 \\
\hline $\begin{array}{l}\text { Mover-se na cadeira de } \\
\text { rodas }\end{array}$ & 6 & 5,8 & 6 & 5,8 & 11 & 10,6 & 69 & 66,3 & 12 & 11,5 \\
\hline Escadas & 32 & 30,8 & 29 & 27,9 & 25 & 24,0 & 11 & 10,6 & 7 & 6,7 \\
\hline
\end{tabular}

No domínio comunicação, a visão foi o item mais afirmaram ter dificuldade ou precisarem de auxílio/ comprometido entre os idosos, sendo que $60(57,7 \%)$ supervisão como pode ser observado na Tabela 4.

Tabela 4 . Distribuição dos idosos restritos ao domicílio segundo o grau de incapacidade no domínio Comunicação. Londrina, 2004.

\begin{tabular}{lcccccccccc}
\hline Atividade & \multicolumn{2}{c}{ Independente } & \multicolumn{2}{c}{$\begin{array}{l}\text { Dificuldade/ } \\
\text { Supervisão/ } \\
\text { Auxílio }\end{array}$} & \multicolumn{2}{c}{$\begin{array}{c}\text { Totalmente } \\
\text { Dependente }\end{array}$} & \multicolumn{2}{c}{ Não se aplica } & \multicolumn{2}{c}{$\begin{array}{c}\text { Taxa de não- } \\
\text { resposta }\end{array}$} \\
& $\mathbf{n}$ & $\mathbf{\%}$ & $\mathbf{n}$ & $\mathbf{\%}$ & $\mathbf{n}$ & $\mathbf{\%}$ & $\mathbf{n}$ & $\mathbf{\%}$ & $\mathbf{n}$ & $\mathbf{\%}$ \\
\hline Visão & 33 & 31,7 & 60 & 57,7 & 9 & 8,6 & - & - & 2 & 1,9 \\
Audição & 75 & 72,1 & 23 & 22,1 & 5 & 4,8 & 1 & 1,0 & - & - \\
Fala & 82 & 78,8 & 16 & 15,4 & 3 & 2,9 & 2 & 1,9 & 1 & 1,0 \\
\hline
\end{tabular}

No domínio Estado Mental, 14 (13,5\%) idosos (12,5\%) na orientação, como pode ser analisado na reportaram dependência em relação à memória e 13 Tabela 5. 
Tabela 5 . Distribuição dos idosos restritos ao domicílio segundo o grau de incapacidade no domínio Estado Mental. Londrina, 2004.

\begin{tabular}{lcccccccccc}
\hline Atividade & \multicolumn{2}{c}{ Independente } & \multicolumn{2}{c}{$\begin{array}{c}\text { Dificuldade/ } \\
\text { Supervisão/ } \\
\text { Auxílio }\end{array}$} & \multicolumn{2}{c}{$\begin{array}{c}\text { Totalmente } \\
\text { Dependente }\end{array}$} & Não se aplica & \multicolumn{2}{c}{$\begin{array}{c}\text { Taxa de não- } \\
\text { resposta }\end{array}$} \\
& $\mathbf{n}$ & $\mathbf{\%}$ & $\mathbf{n}$ & $\mathbf{\%}$ & $\mathbf{n}$ & $\mathbf{\%}$ & $\mathbf{n}$ & $\mathbf{\%}$ & $\mathbf{n}$ & $\mathbf{\%}$ \\
\hline Memória & 48 & 46,1 & 38 & 36,5 & 14 & 13,5 & 2 & 1,9 & 2 & 1,9 \\
Orientação & 64 & 61,5 & 22 & 21,1 & 13 & 12,5 & 1 & 1,0 & 4 & 3,8 \\
\hline
\end{tabular}

No domínio Utilização de Recursos, verificou-se maior dependência nas atividades relacionadas a fazer compras $56(53,8 \%)$ e utilizar o telefone 44
$(42,3 \%)$, porém as demais respostas referentes à dependência merecem realce, por apresentarem-se acima de $30 \%$, como mostra a Tabela 6 .

Tabela 6 . Distribuição dos idosos restritos ao domicílio segundo o grau de incapacidade no domínio: Utilização de Recursos. Londrina, 2004.

\begin{tabular}{lcccccccccc}
\cline { 2 - 10 } \multicolumn{1}{c}{ Atividade } & \multicolumn{2}{c}{ Independente } & \multicolumn{2}{c}{$\begin{array}{c}\text { Dificuldade/ } \\
\text { Supervisão/ } \\
\text { Auxílio }\end{array}$} & $\begin{array}{c}\text { Totalmente } \\
\text { Dependente }\end{array}$ & Não se aplica & $\begin{array}{c}\text { Taxa de não- } \\
\text { resposta }\end{array}$ \\
\cline { 2 - 11 } & $\mathbf{n}$ & $\mathbf{\%}$ & $\mathbf{n}$ & $\mathbf{\%}$ & $\mathbf{n}$ & $\mathbf{\%}$ & $\mathbf{n}$ & $\mathbf{\%}$ & $\mathbf{n}$ & $\mathbf{\%}$ \\
\hline Limpeza da casa & 28 & 26,9 & 16 & 15,4 & 38 & 36,5 & 21 & 20,2 & 1 & 1,0 \\
Preparo alimentos & 43 & 41,3 & 7 & 6,7 & 37 & 35,6 & 16 & 15,4 & 1 & 1,0 \\
Compras & 28 & 26,9 & 6 & 5,8 & 56 & 53,8 & 14 & 13,5 & - & - \\
Lavar roupa & 37 & 35,6 & 7 & 6,7 & 38 & 36,5 & 21 & 20,2 & 1 & 1,0 \\
Uso de telefone & 37 & 35,6 & 14 & 13,5 & 44 & 42,3 & 9 & 8,6 & - & - \\
Uso de transporte & 27 & 26,0 & 15 & 14,4 & 34 & 32,7 & 27 & 26,0 & 1 & 1,0 \\
Uso da Medicação & 45 & 43,3 & 15 & 14,4 & 40 & 38,5 & 4 & 3,8 & - & - \\
Manuseio dinheiro & 51 & 49,0 & 10 & 9,6 & 37 & 35,6 & 6 & 5,8 & - & - \\
\hline
\end{tabular}

\section{Discussão}

Os resultados do presente estudo não podem ser generalizados para toda a população idosa restrita ao domicílio do Município, pois o local pesquisado atende basicamente pessoas com baixa renda ou escolaridade, caracterizando uma amostra homogênea quanto ao aspecto sócio-econômico, conforme dados apresentados pelo PAINP (MESAS et al., 2003).
A revisão de literatura não revelou nenhum estudo que considerasse a capacidade funcional de idosos restritos ao domicílio e permitisse algum tipo de comparação com as informações obtidas neste trabalho. Esse fato indica uma necessidade de estudos de maiores proporções envolvendo idosos restritos ao domicílio, e com amostras que sejam representativas da população em geral, com o devido controle das variáveis sócio-econômicas e outras 
relacionadas ao nível de dependência, estado mental e doenças associadas. Dessa forma, as considerações serão feitas com base em estudos epidemiológicos envolvendo idosos institucionalizados, participantes de grupos de convivência, usuários do serviço público de saúde e residentes na comunidade, mas não necessariamente restritos ao domicílio.

$\mathrm{Na}$ interpretação dos resultados, as informações provenientes das escalas de atividades de vida diária e atividades instrumentais são auto-referidas e, portanto, podem sofrer influência das funções cognitivas, da cultura, da linguagem e da escolaridade. Dessa maneira, erros classificatórios de incapacidade dos idosos podem ter ocorrido em decorrência desses vieses. De acordo com Rosa et al., (2003), as medidas auto-referidas fornecem informações sobre a limitação funcional de idosos em determinado contexto social, as quais dificilmente podem ser obtidas a partir de medidas diretas do desempenho funcional.

No tocante aos dados sóciodemográficos pertinentes aos participantes do estudo, foram investigados o gênero, a idade e o estado civil. Podese observar o predomínio do sexo feminino e tais resultados confirmam a tendência em estudos com idosos (GONÇALVES; DIAS; LIZ, 1999; DERBERT, 1996; PEREIRA; CURIONO; VERAS, 2003; ARAUJO; ALVES, 2000). No Brasil, o número absoluto de mulheres idosas tem sido superior quando confrontado com o de homens de 65 anos ou mais. Essa situação, segundo Berquó (1996), decorre da existência de mortalidade diferencial por sexo que prevalece há muito tempo na população brasileira. $\mathrm{O}$ aumento da expectativa de vida para as mulheres é mais significativo do que para os homens, caracterizando o fenômeno de feminização da população idosa (apud PEREIRA; CURIONO; VERAS, 2003).

Pesquisas realizadas pelo IBGE (PARANÁ, 2004a) demonstram um crescimento acelerado da população brasileira acima dos 80 anos, como foi observado no presente estudo.
Com relação ao estado civil, os viúvos predominaram nessa amostra, com $49,0 \%$, seguido pelos casados, com $43,3 \%$. Considerando que as mulheres idosas constituem o maior número de participantes desse estudo, Chamowicz (1998) justifica o fato pela menor longevidade dos homens, mas, também, pela maior freqüência de recasamento dos homens, após a viuvez, e maior tendência de estes se casarem com mulheres mais jovens.

A população de idosos não alfabetizados foi predominante, seguida dos idosos que não completaram o $1^{\circ}$ grau e os semi-alfabetizados. Este dado sócio-demográfico é relevante considerando os estudos de Donmez, Gokkoca e Dedeoglu (2004) e Rosa (2003), os quais encontraram, respectivamente, chances cerca de 4,5 e 5,0 vezes maior de ter dependência moderada/grave entre os idosos com nível mais baixo de escolarização (apenas lê e escreve/analfabetos).

A maioria dos idosos entrevistados apresentaram renda familiar mensal baixa e de acordo com Kawamoto, Yosihda e Oka (2004) e Rautio, Heikkinen e Heikkinen (2001) existe forte associação entre boa condição financeira e menor incapacidade funcional.

O predomínio de doenças crônico-degenerativas é muito acentuada entre os idosos, como observado no presente estudo. Araújo e Alves (2000) apontam que entre as conseqüências da maior presença destas doenças em idosos destacam-se o maior tempo de internação hospitalar, a recuperação mais lenta e maior freqüência de reinternações e de incapacidades.

A diminuição da capacidade funcional observada em idosos do presente estudo é também encontrada na literatura do Brasil e de outros países. Estudo realizado por Jagger et al. (2001) no Reino Unido verificou uma ordem de restrição de atividades, iniciando-se pelo banho, locomoção, vestir-se, higiene e por fim a alimentação. O estudo longitudinal de Dunlop, Hughes e Manheim (1997) realizado nos Estados Unidos com 5151 idosos revelou a diminuição da capacidade funcional principalmente em relação 
ao banho, higiene, cuidado pessoal, vestir-se e locomoção.

Lucena et al. (2002) afirmaram também que o banho e o vestuário apresentam um maior nível de dependência nos idosos institucionalizados da Paraíba.

Analisando os aspectos relacionados aos domínios AVDs e utilização de recursos, observou-se maior perda das atividades com utilização de recursos em relação às AVDs, confirmando o estudo de Shinkai et al., (2003) no Japão.

A alta taxa de dor na população estudada e a maior dependência nas atividades de utilização de recursos concorda com a afirmação de Dellaroza (2000), em que a pessoa com dor deixa de realizar inicialmente atividades de vida instrumental, mas, apenas nas situações limites, deixa de realizar às AVDs.

\section{Comentários Finais}

Verificou-se que os idosos restritos ao domicílio do Conjunto Habitacional Ruy Virmond Carnascialli mostraram-se dependentes ou necessitarem de cuidados principalmente em relação aos aspectos: banho, cuidado pessoal, higiene, locomoção externa, visão, fazer compras e utilizar o telefone.

A manutenção da capacidade funcional tem importantes implicações para a qualidade de vida dos idosos, por estar relacionada com a capacidade de ocupar-se com o trabalho até idades mais avançadas e/ou com atividades agradáveis. Portanto, é fundamental a implantação de políticas preventivas e de reabilitação efetivas, para a eliminação de fatores de risco relacionados com a incapacidade funcional. Os fatores socioeconômicos e demográficos são pouco sujeitos a intervenções preventivas. Já os programas e serviços podem dar ênfase aos problemas de visão, controle da hipertensão e saúde mental, passíveis de intervenção preventiva. A dependência não é um estado permanente, mas um processo dinâmico cuja evolução pode se modificar e até ser prevenida ou reduzida com a existência de ambiente e assistência adequados.

O presente trabalho revela um diagnóstico de incapacidade funcional apresentado pelos idosos e destaca também, a necessidade de integração dos diferentes profissionais envolvidos nessa questão, cada qual com suas competências específicas e em sintonia de ações de promoção da saúde até o estabelecimento de redes de apoio a cuidados de longa duração na comunidade.

\section{Referências}

ARAUJO, T. C. N.; ALVES, M. I. C. Perfil da população idosa no Brasil. Textos sobre o Envelhecimento, v.3, p.719,2000.

BENNETT, A. Cecil Medicina Interna Básica. 4. ed. Rio de Janeiro: Guanabara Koogan, 1998.

CHAIMOWICZ, F. A saúde dos idosos brasileiros às vésperas do século XXI: problemas, projeções e alternativas. 1996. Disponível em: <http://www.scielo.br/ scielo.> Acesso em: 16 set. 2004.

CHAMOWICZ F. Os idosos brasileiros no século XXI: demografia, saúde e sociedade. Belo Horizonte (MG): PostGraduate; 1998.

CORDEIRO, R. C, DIAS, R. C, DIAS, R. C et al. Concordância entre observadores de um protocolo de avaliação fisioterapêutica em idosas institucionalizadas. Revista de Fisioterapia, São Paulo, v. 9, p.69-77, 2002.

DERBERT GG. As representações (esteriótipos) do perfil do idoso na sociedade atual.In: SEMINÁRIO INTERNACIONAL ENVELHECIMENTO POPULACIONAL: uma agenda para o final do século, 1 ., 1996, Brasília. Anais... Brasília: MPAS/SAS, 1996. p.35-45.

DELLAROZA, M.S.G. Prevalência e caracterização da dor crônica em idosos servidores municipais de Londrina-Pr.2000. Dissertação (Mestrado) - Universidade de São Paulo. Escola de Enfermagem, São Paulo.

DONMEZ, L.; GOKKOCA, Z.; DEDEOGLU, N. Disability and its effects on quality of life among older people living in Antalya city center, Turkey. Archives of Gerontology and Geriatrics, Elsevier, v.40, n.2, p.213-223, mar.-apr., 2005.

DUNLOP, D.D.; HUGHES, S.L.; MANHEIM, L.M. Disability in activities of daily living: patterns of change and a hierarchy of disability. American Journal of Public Health, Boston, v.87, n.3, p.378-383, mar., 1997. 
FEDRIGO, C.R.A.M. Fisioterapia na Terceira Idade - O Futuro de Ontem é Realidade de Hoje. Revista Reabilitar, São Paulo, n.5, p. 18-26, 1999.

GONÇALVES, L.H.T; DIAS, M.M.; LIZ, T.G. Qualidade de vida de idosos independentes segundo proposta de avaliação de Flanagan. O mundo da Saúde ,São Paulo v.23, n.4, p.214-220, jul/ago., 1999.

HÉBERT, R. Functional decline in old age. Canadian Medical Association Journal, Ottawa, v.157, n.8, p.10371045, Oct. 1997.

HÉBERT, R.; GUILBAULT, J.; DESROSIERS, J.; DUBUC, $\mathrm{N}$. The functional autonomy measurement system (SMAF): a clinical based instrument for measuring disabilities and handicaps in older people. Journal of the Canadian Geriatrics Society, Ottawa, v.4, n.3, set. 2001.

JAGGER, C.; ARTHUR, A. J.; SPIERS, N. A.; CLARKE, M. Patterns of Onset of Disability in Activities of Daily Living with Age. American Geriatrics Society, New York, v.49, n. 4, p.404-409, 2001.

LUCENA, N. M. G.; GUERRA, R. O.; LUCENA, A. B.; SILVA, C. F.; NASCIMENTO, R. Q. Análise da capacidade funcional em uma população geriátrica institucionalizada em João Pessoa. Fisioterapia Brasil, Rio de Janeiro, v.3, n.3,p.164-169, 2002.

MESAS, A. E., DELLAROZA, M. S., PERDIGÃO, S. D., CABRERA, M. A. S., YAMADA, K., TRELHA, C. S. Auto percepção da saúde em idosos da comunidade. In: CONGRESSO PAULISTA DE GERIATRIA E GERONTOLOGIA, 3., 2003, Santos. Anais...Santos, 2003.

KAWAMOTO, R.; YOSHIDA, O.; OKA, Y. Factors related to functional capacity in community-dwelling elderly. Geriatrics and Gerontology Internacional, Tokyo, v.4, p.105-110, 2004.

PARANÁ. Secretaria do Estado da Saúde. Instituto de Saúde do Paraná. Manutenção da Capacidade Funcional. Disponível em: <http://www.saude.pr.gov.br/ Sesa_fazendo/Saude_idoso/capacidade_funcional.htm $>$. Acesso em: 4 ago. $2004 \mathrm{a}$.
PARANÁ. Secretaria do Estado da Saúde. Conselho Estadual dos Direitos do Idoso (CEDI). Perfil do Idoso do Estado do Paraná. Disponível em: <http://www.saude. pr.gov.br/saudeidoso/> Acesso em: 4 ago. 2004b.

PEREIRA, R. S.; CURIONO, C.C.;VERAS, R. Perfil demográfico da população idosa no Brasil e no Rio de Janeiro em 2002. Textos Envelhecimento, Rio de Janeiro, v.6, n.1, 2003.

RAMOS L.R. Fatores determinantes do envelhecimento saudável em idosos residentes em centro urbano: Projeto Epidoso, São Paulo. 2003. Disponível em: < http:// www.scielosp.org/scielo>. Acesso em: 21 ago. 2004.

RAMOS, L.R.; ROSA, T. E. C.; OLIVEIRA, Z. M.; MEDINA, M. C. G.; SANTOS, F. R. G. Perfil do idoso em área metropolitana na região sudeste do Brasil: resultado de inquérito domiciliar. Revista de Saúde Pública, São Paulo, v.27, n.2, p.87-94, 1993.

RAUTIO, N.; HEIKKINEN, E.; HEIKKINEN, R.L. The association of socio-economic factors with physical and mental capacity in elderly men and women. Archives of Gerontology and Geriatrics, Elsevier, v. 33, p.163-178, 2001.

ROSA, T. E. da C.; BENÍCIO, M. H. D.; LATORRE, M. do R. D. de O.; RAMOS, L. R. Fatores determinantes da Capacidade Funcional entre idosos. Revista Saúde Pública; São Paulo, v.37, n.1, fev. 2003.

SHINKAI, S.; KUMAGAI, S.; FUJIWARA, Y.; AMANO, H.; YOSHIDA, Y.; WATANABE, S.; ISHIZAKI, T.; SUZUKI, T.; SHIBATA, H. Predictors for the onset of functional decline among initially non-disabled older people living in a community during a 6-year follow-up. Geriatrics and Gerontology Internacional, Tokyo, 3: S31S39, 2003.

SOARES, J. F.; SIQUEIRA A. L. Introdução à estatística médica. Belo Horizonte: UFMG, 1999.

SOUZA, J.A. G.; IGLESIAS, A.C.R.G. Trauma no Idoso. Revista Associação Médica Brasileira, São Paulo, v.48, n.1,p.79-86, 2002. 